\title{
Stark widths of faint Si II lines
}

\author{
A. Lesage ${ }^{1}$ and R. Redon ${ }^{2}$ \\ 1 GEPI - UMR 8111, Observatoire de Paris-Meudon, 92125 Meudon Cedex, France \\ ${ }^{2}$ Laboratoire d'Optique Appliquée, Université de Toulon et du Var, 83957 La Garde, France
}

Received 28 October 2003 / Accepted 29 January 2004

\begin{abstract}
Line profiles of faint Si II lines of astrophysical interest are observed in a conventional shock tube. Electron density is $1.3 \times 10^{23} \mathrm{~m}^{-3}$, and the temperature is $11000 \mathrm{~K}$. The Stark effect is the dominant broadening mechanism. The Si II line at $\lambda 419.07 \mathrm{~nm}$ (multiplet 7.26, Moore 1965) Stark width is measured and compared with theoretical prediction.
\end{abstract}

Key words. atomic data - line: profiles - plasmas - stars: atmospheres

\section{Introduction}

Because of the large cosmic abundance of silicon, Si II spectra are of special importance in solar and stellar studies. For stars with effective temperature from $10000 \mathrm{~K}$ to $20000 \mathrm{~K}$ (i.e. A0 to B3) the Si II spectrum is dominant with strong lines in the visible. The Ap-Si stars can be enriched in silicon up to a factor of 100 (compared with the sun). In such stars the spectral analysis of that element becomes more critical. Several problems are specific to such classes of stars. One of them is the depressions of the continuum in the visible and in the UV. This problem has been analyzed and partially solved around $140 \mathrm{~nm}$ (Artru et al. 1981a,b), but not yet at 420, 520 and $630 \mathrm{~nm}$ (Artru et al. 1981b; Artru 1986; Artru \& Lanz 1987). The second and third problems are the silicon abundance determination in their atmosphere (Lanz \& Artru 1988) and the calculation of their synthetic spectra. Despite a thorough compilation of the oscillator strengths of Si II done by Lanz \& Artru 1985, the abundance problem has not yet been completely solved. The abundance remains a problem due the fact that $30 \%$ of the oscillator strengths are still unknown. The third problem occurs because of the lack of knowledge of Si II Stark parameters. These are needed when in stellar atmospheres, the Stark effect becomes larger than the Doppler effect (Lanz \& Artru 1988). In such a case, the Stark width contributes to the de-saturation of the line core. Then the equivalent width of stellar absorption lines strongly depends on the Stark effect. This is also the case of very strong lines where most of the absorbed energy resides in the wings of the Voigt profile beyond the saturation plateau. Here, the equivalent width can be determined by Stark broadening only (Lanz et al. 1988; Lanz et al. 1988; Lesage 1995). From the compilation (Lanz et al. 1985) of calculated and

Send offprint requests to: A. Lesage, e-mail: alain.lesage@obspm.fr measured Stark parameters published at that time, the authors conclude that more than half of the Si II multiplets of astrophysical interest have no published Stark parameters. The ones that they have adopted for their own Ap-Si star spectra interpretation were those calculated by the semi-classical method. Their accuracy can be estimated as being $20 \%$ for the transition having lower energy levels (Mult. 1 to 7). A greater uncertainty is expected for lines arising from higher levels, which are prone to greater broadening. It is the purpose of the present article to show what can be observed with a very large aperture luminous spectrograph and measured with an unfolding procedure, specially designed for faint lines and low-resolution spectral observation. The relative size between astronomical and laboratory plasma sources explains by itself the encountered difficulties.

\section{Experimental}

\subsection{Introduction}

Starting with Table 1 published by Lanz et al. (1988) concerning the 19 selected visible multiplets of astrophysical interest, we provided our own data (Table 1), which describes the experimental and theoretical data presently available. The table shows that apart from the lower-lying multiplets 1 to 5 , multiplets 7.03 and 23 only have measured values which can be compared to the calculated ones. In that comparison, we retain the values provided by Griem's semi-empirical formula (Griem 1968) because in the case of Si II these values are in better agreement with experiment than with Griem's semi-classical calculations (1974), and those of Dimitrijevic using the method developed by Sahal-Bréchot (1969a,b). Table 1 shows also that in spite of the recent work by Gonzalez (Gonzalez et al. 2001; Gonzalez 1999) there are still eleven multiplets without measured values. 
Table 1. Stark widths of singly-ionized silicon lines of astrophysical interest. Columns 1 to 4 list the ionized silicon lines of astrophysical interest. Column 5 shows: previous measured values for mults. 1 to 7.03 and 23, the presently observed faint Si II lines (mults. 7.05 to 7.16 and 23) and the width value of the presently measured mult. 7.26 lines. Columns 6 and 7 show a selection of calculated values done on the basis of best agreement with experiment. Abbreviations: L for Lesage, G for Gonzalez, W for Wollschlager, D for Dimitrijevic, LS for Lesage. Corresponding data are in Lesage et al. (1983), Wollschlager et al. (1997), Lanz et al. (1988), and Lesage et al. (1977).

\begin{tabular}{|c|c|c|c|c|c|c|}
\hline $\begin{array}{c}\text { Mult } \\
\mathrm{N}^{\circ}\end{array}$ & $\begin{array}{c}\text { Transition } \\
\text { Moore } 1965\end{array}$ & $\begin{array}{c}\lambda \\
(\mathrm{nm})\end{array}$ & $\begin{array}{c}\text { Intensity } \\
\text { Striganov } \\
\text { \& Sventitskii } \\
1966\end{array}$ & $\begin{array}{c}\Delta \lambda \mathrm{nm} \\
\text { Measurements } \\
N_{\mathrm{e}}=10^{23} \mathrm{~m}^{3}\end{array}$ & $\begin{array}{c}\Delta \lambda \mathrm{nm} \\
\text { Calculation } \\
N_{\mathrm{e}}=10^{23} \mathrm{~m}^{3} \\
\text { Griem } 1968\end{array}$ & $\begin{array}{c}\Delta \lambda \mathrm{nm} \\
\text { Calculation } \\
N_{\mathrm{e}}=10^{23} \mathrm{~m}^{3} \\
\text { Sahal 1969a,b }\end{array}$ \\
\hline 1 & $3 p^{2} \mathrm{D}-4 \mathrm{p}^{2} \mathrm{P}^{0}$ & $\begin{array}{l}385.602 \\
386.260 \\
385.366\end{array}$ & $\begin{array}{l}500 \\
200 \\
100\end{array}$ & $\begin{array}{l}\mathrm{L} .064 \text { at } 16000 \mathrm{~K} \\
\mathrm{G} .050 \text { at } 18000 \mathrm{~K} \\
\mathrm{~W} .053 \text { at } 14000 \mathrm{~K}\end{array}$ & .059 at $10^{4} \mathrm{~K}$ & LS .050 at $10^{4} \mathrm{~K}$ \\
\hline 2 & $4 s^{2} S-4 p^{2} P^{o}$ & $\begin{array}{l}634.710 \\
637.136\end{array}$ & $\begin{array}{l}1000 \\
1000\end{array}$ & $\begin{array}{l}\mathrm{L} .124 \text { at } 16000 \mathrm{~K} \\
\mathrm{G} .10 \text { at } 18000 \mathrm{~K}\end{array}$ & .179 at $10^{4} \mathrm{~K}$ & LS .171 at $10^{4} \mathrm{~K}$ \\
\hline 3 & $3 d^{2} D-4 f^{2} F^{o}$ & $\begin{array}{l}413.089 \\
412.807\end{array}$ & $\begin{array}{l}500 \\
300\end{array}$ & $\begin{array}{l}\mathrm{L} .10 \text { at } 16000 \mathrm{~K} \\
\mathrm{G} .10 \text { at } 18000 \mathrm{~K}\end{array}$ & .120 at $10^{4} \mathrm{~K}$ & LS .099 at $10^{4} \mathrm{~K}$ \\
\hline 3.01 & $3 d^{2} D-5 p^{2} P^{0}$ & $\begin{array}{l}407.5451 \\
407.6781 \\
407.2711\end{array}$ & $\begin{array}{c}20 \\
15 \\
3\end{array}$ & & & D .155 at $10^{4} \mathrm{~K}$ \\
\hline 4 & $4 p^{2} P^{0}-5 s^{2} S$ & $\begin{array}{l}597.8929 \\
595.7561\end{array}$ & $\begin{array}{l}500 \\
500\end{array}$ & $\begin{array}{l}\mathrm{L} .312 \text { at } 16000 \mathrm{~K} \\
\mathrm{G} .27 \text { at } 16000 \mathrm{~K}\end{array}$ & .281 at $10^{4} \mathrm{~K}$ & LS .283 at $10^{4} \mathrm{~K}$ \\
\hline 5 & $4 p^{2} P^{0}-4 d^{2} D$ & $\begin{array}{l}505.5981 \\
504.1026 \\
505.6314\end{array}$ & $\begin{array}{c}1000 \\
1000 \\
30\end{array}$ & $\begin{array}{l}\mathrm{L} .21 \text { at } 16000 \mathrm{~K} \\
\mathrm{G} .254 \text { at } 18000 \mathrm{~K}\end{array}$ & .233 at $10^{4} \mathrm{~K}$ & LS .262 at $10^{4} \mathrm{~K}$ \\
\hline 6 & $4 p^{2} P^{0}-6 s^{2} S$ & $\begin{array}{l}333.9819 \\
333.3139\end{array}$ & $\begin{array}{l}500 \\
300\end{array}$ & & & D. .176 at $10^{4} \mathrm{~K}$ \\
\hline 7 & $4 p^{2} P^{0}-5 d^{2} D$ & $\begin{array}{l}321.0025 \\
320.3872\end{array}$ & $\begin{array}{l}200 \\
100\end{array}$ & & & D .222 at $10^{4} \mathrm{~K}$ \\
\hline 7.02 & $4 d^{2} D-5 f^{2} F^{0}$ & $\begin{array}{l}784.972 \\
784.880\end{array}$ & $\begin{array}{l}500 \\
400\end{array}$ & $\mathrm{G} .439$ at $18000 \mathrm{~K}$ & & D 1.47 at $2 \times 10^{4} \mathrm{~K}$ \\
\hline 7.03 & $4 d^{2} D-6 f^{2} F^{0}$ & $\begin{array}{l}546.6868 \\
546.6432\end{array}$ & $\begin{array}{l}500 \\
500\end{array}$ & G.95 at $18000 \mathrm{~K}$ & & D 1.64 at $2 \times 10^{4} \mathrm{~K}$ \\
\hline 7.05 & $4 d^{2} D-7 f^{2} F^{o}$ & $\begin{array}{l}462.1721 \\
462.1418\end{array}$ & $\begin{array}{l}150 \\
100\end{array}$ & Presently observed & & D 2.33 at $10^{4} \mathrm{~K}$ \\
\hline 7.06 & $4 d^{2} D-8 f^{2} F^{o}$ & $\begin{array}{l}420.0898 \\
420.0657\end{array}$ & $\begin{array}{l}40 \\
30\end{array}$ & Presently observed & & D 3.45 at $10^{4} \mathrm{~K}$ \\
\hline 7.07 & $4 d^{2} D-9 f^{2} F^{o}$ & $\begin{array}{l}395.4507 \\
395.4296\end{array}$ & $\begin{array}{c}10 \\
5\end{array}$ & & & D 5.26 at $10^{4} \mathrm{~K}$ \\
\hline 7.13 & $4 \mathrm{f}^{2} \mathrm{~F}^{\mathrm{o}}-6 \mathrm{~g}^{2} \mathrm{G}$ & 623.9630 & 100 & Presently observed & & D 3.22 at $10^{4} \mathrm{~K}$ \\
\hline 7.14 & $4 \mathrm{f}^{2} \mathrm{~F}^{\mathrm{o}}-7 \mathrm{~g}^{2} \mathrm{G}$ & 518.5535 & 100 & Presently observed & & D 5.31 at $10^{4} \mathrm{~K}$ \\
\hline 7.15 & $4 f^{2} F^{o}-8 g{ }^{2} G$ & 467.3273 & 20 & Presently observed & & D 8.47 at $10^{4} \mathrm{~K}$ \\
\hline 7.16 & $4 f^{2} F^{o}-9 g{ }^{2} G$ & 437.6957 & 5 & Presently observed & & D 13.1 at $10^{4} \mathrm{~K}$ \\
\hline 7.26 & $3 \mathrm{~d}^{\prime 2} \mathrm{D}^{0}-4 \mathrm{p}^{\prime 2} \mathrm{P}$ & $\begin{array}{l}419.0724 \\
419.8133 \\
418.3345\end{array}$ & $\begin{array}{c}100 \\
50 \\
10\end{array}$ & $\begin{array}{l}\text { Present measurement: } \\
.095 \text { at } T=11000 \mathrm{~K}\end{array}$ & & D .051 at $10^{4} \mathrm{~K}$ \\
\hline 23 & $4 \mathrm{p}^{\prime}{ }^{4} \mathrm{D}-4 \mathrm{~d}^{\prime}{ }^{4} \mathrm{~F}^{0}$ & 520.2413 & 500 & $\begin{array}{c}\mathrm{G} .133 \text { at } T=18000 \mathrm{~K} \\
\text { Presently observed }\end{array}$ & & D .124 at $2 \times 10^{4} \mathrm{~K}$ \\
\hline
\end{tabular}

\subsection{Plasma production}

The plasma is generated in a diaphragm shock tube $(52 \times 52$ $\mathrm{mm})$. Strong ionizing shock waves are achieved by a condenser discharge $(60 \mu \mathrm{F}, 30 \mathrm{kV})$ through the driver gas $\left(6 \times 10^{5} \mathrm{~Pa}\right)$, of $\mathrm{H}_{2}$. The test gas is neon (700-800 Pa) plus a small admixture $(0.45-3.5 \%)$ of Tetramethylsilane (TMS). Temperatures from 
11000 to $14500 \mathrm{~K}$ and electron densities from 0.5 to $2.0 \times$ $10^{23} \mathrm{~m}^{-3}$, are achieved in the radiation cooled region behind the reflected shock wave.

\subsection{Interferometry}

Electron densities are determined with a two-wavelength (488.0 nm, $632.8 \mathrm{~nm}$ ), two-phase Michelson interferometer (Meiners \& Weiss 1973) with photoelectric detection. The interferometer has been improved by using oscillating mirrors in the reference arm and by digital recording of the interferograms (Fervers 1980). For electron density measurements, the interferometer's fringes have to be traced through the incident and reflected shock front and the relaxation region until LTE is achieved, where the silicon spectra are then observed. For that purpose, the distance between the interferometer beam and the reflecting wall had to be adjusted within the narrow interval of 3.0-3.5 mm. As in the previous investigation (Meiners et al. 1973), the thin boundary layers are neglected.

\subsection{Spectroscopy}

Line profiles are observed with two different spectrographs and recording systems. The first is a large aperture (F: 1.7) luminous spectrograph with an intensified RETICON detector in the focal plane. The large aperture is obtained by two mirrors, as for the Cassegrain telescope, but used in the reverse way for the light. Two large surface $(135 \times 220.5 \mathrm{~mm})$ gratings $(3600$ and 2230 grooves $/ \mathrm{mm}$ ) enable the 350 to $530 \mathrm{~nm}$ and 500 to $600 \mathrm{~nm}$ spectral ranges to be covered. The resolution is limited by the $25 \mu \mathrm{m}$ size of the detector diodes. The exposure time is controlled by a gating pulse applied to the intensifier. The detector's linearity is checked with a light source emitting a continuous spectrum (tungsten ribbon) and a series of neutral density filters of known transmission. In the range of intensity of the observed lines, the linearity of the detector is within $2 \%$.

As the unfolding procedure assumes that the observed line shapes do not vary versus the position or the intensity of the line on the detector, that behavior is checked with a narrow iron line (low pressure discharge), which is also used to measure the apparatus function.

The second device is a 24-channel polychromator with optical fibers, photomultipliers and a digital data acquisition system, which records the $\mathrm{Ne} \mathrm{I} \lambda=585.2 \mathrm{~nm}$ line profile every microsecond (Meiners \& Schönartz 1983). The spectral distance between adjacent channels is $0.032 \mathrm{~nm}$. A series of profiles is observed during the plasma life-time, every one belonging to a specific value of the electron density, which is known from interferometry. A PTB calibrated tungsten ribbon lamp is used to measure the plasma radiance absolutely. A description of the procedure and a discussion of the linearity can be found in Zangers \& Meiners (1989). The plasma temperature is measured on the basis of the absolute intensity of the Ne I line $\lambda=585.2 \mathrm{~nm}$, with $A_{\mathrm{ki}}=0.682 \times 10^{8} \mathrm{~s}^{-1}$ [CRC handbook].

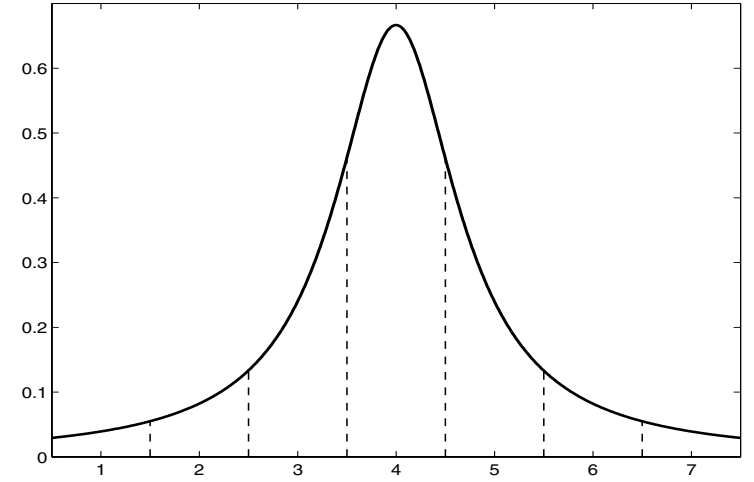

Fig. 1. Real profile of a spectral line shown with a division corresponding to seven adjacent detector pixels.

\section{Data reduction}

\subsection{Doppler effect, self-absorption and intensity calibration}

The Doppler width is calculated for the measured plasma temperature $T=11000 \mathrm{~K} ; \Delta_{\mathrm{D}}=.0059 \mathrm{~nm}$. The faint lines intensity from the obtained spectra can be directly compared to the one of the nearby Si II (Mult. 3) strong lines. For the spectra where Si II (Mult. 7.26) line profiles were measured the strong line intensity saturated the detector. For another spectra, not retained for measurement, taken in very similar physical conditions, the intensity of the $419.07 \mathrm{~nm} \mathrm{Si}$ II (Mult. 7.26) line is 70 times smaller than the $413.09 \mathrm{~nm} \mathrm{Si} \mathrm{II} \mathrm{(Mult.} \mathrm{3)} \mathrm{line.}$ Consequently, the absorption of light at the peak of the faint lines is unlikely and has been neglected in the calculations.

The intensity calibration of the detector, spectrograph and associated optical system is checked with a source emitting a continuous spectrum (tungsten ribbon lamp at $T=2856 \mathrm{~K}$ ). The variation of transmission and sensitivity is taken into account in the calculations.

\subsection{Unfolding procedure}

Classically, when the spectral model is compared to the one obtained via a diode array detector, it is understood that the integrated light on a pixel is a good approximation for the mean value across the detector pixel. This is true when there are enough pixels along the line profile, especially when compared to its half width. But in the present case, due to the choice of a large aperture and a rather low dispersion of the spectrograph, the line profiles cover only a limited numbers of pixels of the OMA detector. The widths at half maximum of the apparatus function and of the silicon lines are of the order of 3 and 5 pixels widths respectively. Consequently the observed profile is distorted compared to the real one. This is due to the fact that a pixel provides the mean value of the intensity of the light regardless of its variation along the width of the pixel. In the present case, we could have an overestimation of the profile width (Figs. 1, 2).

To make allowance for this effect a new procedure has been enacted. The spectral model used in the unfolding procedure is calculated in such a way that it can be directly compared to 


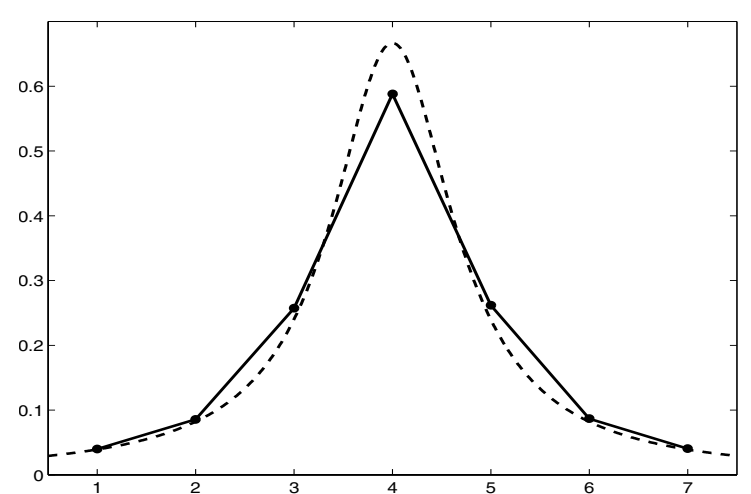

Fig. 2. Signals $(\bullet)$ aquired by the OMA system compared to the real line profile (- - - ). In such a case, full width at half maximum is overestimated.

the observed spectra. The broadening due to the Stark effect is depicted by a Lorentzian lineshape:

$f_{\mathrm{S}}(\lambda)=\frac{1}{\Delta \lambda_{\mathrm{S}}}\left(\frac{\lambda_{0}}{\lambda}\right)^{2} \frac{1}{1+4\left(\frac{\lambda_{0}\left(\lambda-\lambda_{0}\right)}{\lambda_{0} \Delta \lambda_{\mathrm{D}}}\right)^{2}}$

the Doppler broadening by a Gaussian function:

$f_{\mathrm{D}}(\lambda)=2 \sqrt{\frac{\ln 2}{\pi \Delta \lambda_{\mathrm{D}}}}\left(\frac{\lambda_{0}}{\lambda}\right)^{2} \exp \left[-4 \ln 2\left(\frac{\lambda_{0}\left(\lambda-\lambda_{0}\right)}{\lambda_{0} \Delta_{\mathrm{D}}}\right)^{2}\right]$

where parameters $\Delta \lambda_{\mathrm{S}}$ and $\Delta \lambda_{\mathrm{D}}$ are Stark and Doppler widths, respectively. The width due to the spectrograph itself is in the present case represented by the following analytical function similar to a Gaussian profile centered at $x_{0}$,

$f_{\mathrm{A}}(x)=\exp \left[-\frac{\left(x-x_{0}\right)^{2}}{4 \ln 2\left(a+\left(\frac{b\left(x-x_{0}\right)}{\left(x-x_{0}\right)^{2}+c}\right)^{2}\right)}\right]$

where the profile width depends on the parameters $a, b$ and $c$. After calibration, the wavelength $\lambda$ corresponds to the variable $x$, and $\lambda_{0}$ to the parameter $x_{0}$. These three profiles are convoluted in one: $r_{i}(\lambda)$ centered on $\lambda_{0}^{(i)}$, having intensity $H_{i}$ and Stark and Doppler widths $\Delta \lambda_{\mathrm{S}}$ and $\Delta \lambda_{\mathrm{D}}$ respectively.

$r_{i}(\lambda)=\left(f_{\mathrm{A}} \otimes f_{\mathrm{D}} \otimes f_{\mathrm{S}}\right)(\lambda)$

where $\otimes$ is the convolution operator.

In the present case of the Si II multiplet 7.26, there are three lines observed and modeled at the same time. Consequently, the synthetized spectrum to be compared with the observed one is the sum $s(\lambda)$ of each one centered at their respective wavelength $\lambda_{0}^{(i)}$ :

$s(\lambda)=\sum_{i=1}^{n} r_{i}(\lambda)$

The spectral model $s(x)$ is convoluted with the spatial integration function $\Pi_{n}(x)$ as follows:

$s_{c}(x)=\left(s \otimes \Pi_{n}\right)(x)$

where:

$\Pi_{n}(x)=\left\{\begin{array}{ll}1, & \text { if }|x| \leq n / 2 \\ 0, & \text { if }|x|>n / 2\end{array}\right.$.
This function models the light integration performed by $n$ subpixels (the real pixel width is divided by $n$ ). When the spectrum received by the detector is considered, the variable $\lambda$ is replaced by the variable $x$. Finally, the $s_{c}(x)$ function is multiplied by a collection of Dirac distributions $\psi_{n}$ where $n$ is also the sampling interval:

$s_{\mathrm{e}}(x)=\left(s_{c} . \Psi_{n}\right)(x)$

where:

$\Psi_{n}(x)=\sum_{k=-\infty}^{\infty} \delta(x-k n)$.

Notice that the $s_{\mathrm{e}}(x)$ spectra tends to $s(x)$ as the number of pixels covered by the line profile increases.

To complete the spectral model before comparison with the observed one, a second-degree polynomial is added to depict the continuum and the nearby line wings of the strong $\mathrm{Si}$ II lines. The associated parameters are calculated on the basis of real data points located outside the multiplet wavelength range.

The fitting algorithm is the Simplex method minimizing the scatter between the spectral model $s_{\mathrm{e}}(x)$ and the experimental one, thanks to slight variations around predetermined values of $\lambda_{0}^{(1)}, H_{i}, \Delta \lambda_{s}$ and the coefficients $c_{i}$ of second-degree polynomial.

\section{Results and discussion}

Twenty experiments have been performed in a variety of physical conditions in order to optimize the faint silicon lines. The slit width was varied from 50 to $175 \mu \mathrm{m}$ without the loss of resolution, filling pressure of neon from 41 to 57 Torr, percentage of TMS from 0.35 to $0.56 \%$, as well as the wave speed, the width and delay of the gate pulse on the detector. Lines of the 7.05, 7.06, 7.13, 7.14, 7.15, 7.16, 7.26 and $23 \mathrm{Si}$ II multiplets have been observed, but the lines of multiplet 7.26 only were of signal-to-noise ratio high enough to be measured. Anomalies in the continuum have not been observed.

The improved method of modeling that takes into account the distortion of the spectral line profile due to the finite width of the detector pixels is applied to the brightest Si II (Mult. 7.26) profile obtained in the present experiment. A very good fit is obtained for the modeled spectra to the observed one (Fig. 3). The obtained full width at half-maximum for the two Si II 419.07 and $419.81 \mathrm{~nm}$ lines is $0.130 \mathrm{~nm}$ at $N_{\mathrm{e}}=1.36 \times 10^{23} \mathrm{~m}^{-3}$ i.e. $0.095 \mathrm{~nm}$ at $N_{\mathrm{e}}=10^{23} \mathrm{~m}^{-3}$ and $T=11000 \mathrm{~K}$ to be compared to the value of $0.051 \mathrm{~nm}$ calculated by Dimitrijevic (Lanz et al. 1988) at $T=10000 \mathrm{~K}$.

This line width measurement precision is basically affected by the deconvolution and the determination of the continuum level methods. In our particular case, the apparatus function shape introduces the larger uncertainty in the calculations, while the baseline position has been determined by two different methods providing reliable results. The estimated error for the reconstructed profile is estimated to be $10 \%$ of the width. The Doppler correction adds $0.2-0.4 \%$ error. The error 




Fig. 3. Si II Multiplet 7.26 lines at 419.07, 419.81 and 418.33 nm: modeled (—), registered (•) spectra, background (. . ) ).

due to the optical depth neglecting is estimated to be a maximum of one percent. Temperature and electron density measurements are determined with $10 \%$ and $5 \%$ accuracies, respectively. These uncertainties combined, according to the Taylor \& Kuyatt 1994 recommendations (root of the sum of the squares of the individuals contributions), add up to $16 \%$.

The present experimental value is 1.9 times larger than the theoretical one, as calculated by Dimitrijevic (in Lanz et al. 1988) according to Sahal-Bréchot's semi-classical predictions (Sahal-Bréchot 1969a,b). But such calculations are not strictly applicable in the case of the present transition between high levels. The quadratic Stark effect is no longer valid, and the linear Stark effect is not yet applicable.

Wilke (2003) has recently measured Stark parameters and transition probabilities of $20 \mathrm{Si}$ II lines in a laser produced plasma, at $N_{\mathrm{e}}=4.8 \times 10^{23} \mathrm{~m}^{-3}$ and $T=21000 \mathrm{~K}$. For the Si II 7.26 multiplet, his measured width is $0.052 \mathrm{~nm}$. The width calculated by Dimitrijevic at $T=20000 \mathrm{~K}$ is even narrower: $0.040 \mathrm{~nm}$. But the variation of the Stark parameter versus temperature is not always as $T^{-0.5}$, as shown in Ralchenko et al. (2003). Consequently, as in the present case the exponent of $T$ is unknown, so the comparison between the present measurements and those of Wilke is hypothetical.

\section{Conclusion}

The series of twenty experiments performed with a very large aperture luminous spectrograph and an intensified cooled detector shows that weak lines of astrophysical interest can be observed with a fully instrumented shock tube as the spectroscopic light source. A new unfolding method specially conceived for the present case, where line profiles cover a limited number of pixels of the detector, provides the first accurate measurement of such a faint line.

\section{References}

Artru, M. C., Jamar, C., Petrini, D., \& Praderie, F. 1981a, A\&AS, 44, 171

Artru, M. C., Jamar, C., Petrini, D., \& Praderie, F. 1981b, A\&A, 96, 380

Artru, M. C. 1986, A\&A, 168, L5

Artru, M. C., \& Lanz, T. 1987, A\&A, 182, 273

Ferfers, H. 1980, Dissertation, Universität Düsseldorf

Gonzalez, V. R. 1999, Ph.D. Thesis, Universidad de Valladolid

Gonzalez, V. R., Aparicio, J. A., del Val, J. A., \& Mar, S. 2001, in Spectral Line Shape 11, ed. J. Seidel, AIP Conf. Proc., 169, 559

Griem, H. R. 1968, Phys. Rev., 165, 258

Griem, H. R. 1974, Spectral Line Broadening by Plasmas (New York: Academic Press)

Lanz, T., \& Artru, M. C. 1988, in Workshop on Elemental Abundance Analyses, Lausane Sept. 1987, ed. S. J., Adelman, \& T. Lanz (Institut d'Astronomie de l'Université de Lausanne)

Lanz, T., \& Artru, M. C. 1985, Phys. Scr., 32, 115

Lanz, T., Dimitrijevic, M. S., \& Artru, M. C. 1988, A\&A, 192, 249

Lesage, A., Sahal-Bréchot, S., \& Miller, M. H. 1977, Phys. Rev. A, 16,1617

Lesage, A., Rathore, B. A., Lakicevic, I. S., \& Puric, J. 1983, Phys. Rev. A, 28, 2264

Lesage, A. 1995, in Astrophysical Applications of Powerful New Databases, ed. S. J. Adelman, \& W. L. Wiese, ASP Conf. Ser., 78,161

Meiners, D., \& Weiss, C. O. 1973, Z. Naturf., 28, 8

Meiners, D., \& Schönartz, N. 1983, in Proc. ICPIG XVI, 644

Moore, C. E. 1965, US Nat. Bur. Stds., NSRDS-NBS 3 Sect. 1

Ralchenko, Yu. V., Griem, H. R., \& Bray, I. 2003, J. Quant. Spectros. Radiat. Transf., 81, 371

Sahal-Bréchot, S. 1969a, A\&A, 1, 91

Sahal-Bréchot, S. 1969b, A\&A, 2, 322

Striganov, A. R., \& Sventitskii, N. S. 1966, Tables of Spectral lines: Neutral and Ionized Atoms (Moscow: Atomizdat)

Taylor, B. N., \& Kuyatt, C. E. 1994, NIST Tech. Note 1297 (Washington, DC: US Government Printing Office)

Wilke, R. 2003, Dissertation, Universität Düsseldorf

Zangers, J., \& Meiners, D. 1989, J. Quant. Spectros. Radiat. Transf., 42,25 